\title{
Análisis de las Prácticas Evaluativas Inclusivas de Tres Docentes de Educación Preescolar en México
}

\author{
Analysis of Inclusive Evaluation Practices of Three Preschool \\ Education Teachers in Mexico
}

\author{
Claudia Isabel Martínez Zapata ${ }^{1 *}$
} Ismael García Cedillo²

${ }^{1}$ Universidad Mesoamericana. ${ }^{2}$ Universidad Autónoma de San Luis Potosí

\begin{abstract}
Las prácticas evaluativas inclusivas se refieren a cómo se instrumentaliza el proceso de evaluación, e incluye las nociones de las docentes con respecto a qué es evaluar, instrumentos que utilizan, tipo de evidencias que presentan, forma de reportar los resultados, uso que dan a los mismos, papel del alumno y la educadora en este proceso y modalidades de evaluación. Se presenta una investigación cualitativa sobre las prácticas que realizan tres educadoras de un Jardín de Niños público en México, cuyo objetivo fue identificar sus prácticas evaluativas inclusivas. Se buscó dar respuesta a la pregunta ¿cuáles son las características de las prácticas evaluativas de las educadoras participantes? Se encontró que las educadoras tienen claro conceptualmente cómo aplicar la evaluación inclusiva, no obstante la implementación de este enfoque en la práctica no ha resultado ser una tarea fácil, en especial por las tensiones que imponen las exigencias de las autoridades educativas.

Descriptores: Educación preescolar, Evaluación de la educación, Evaluación del docente, Método de evaluación.
\end{abstract}

\begin{abstract}
A key aspect of inclusive education is the process of evaluation. This article presents a qualitative research on the practices of three preschool teachers of a public Kindergarten in Mexico, in order to identify their inclusive evaluative practices. Assessment inclusive practices refers to how the evaluation process is instrumentalized, including the educator's notions regarding the meaning of the evaluation process, instruments used, type of evidence presented, how to report the results of the evaluation, how they use the results thereof, the role of the student and the teacher and the assessment procedures. It was found that teachers have clear notions about how to apply formative inclusive assessment; however, the implementation of this approach in practice has not been an easy task, especially for the tensions imposed by the requirements of the educative authorities.
\end{abstract}

Keywords: Preschool education, Educational evaluation, Teacher evaluation, Evaluation methods. 


\section{Introducción}

La evaluación es un componente muy importante del proceso educativo, es pieza clave para que el alumno desarrolle sus habilidades, pues a partir de la evaluación las educadoras toman decisiones para ajustar su práctica a fin de mejorar el aprendizaje de sus alumnos. En $\mathrm{México}^{1}$, en el programa actual de 2011, hay una propuesta de evaluación que parece estar muy acorde a los propósitos de la educación en general y de la educación inclusiva en particular. Sin embargo, no hay investigaciones publicadas que indiquen si las educadoras están atendiendo las sugerencias del mencionado programa o si sus procesos de evaluación son inerciales. Por consiguiente, el propósito de este artículo es dar cuenta del tipo de prácticas evaluativas que las educadoras ${ }^{2}$ participantes realizan con sus alumnos, se busca identificar si dichas prácticas concuerdan con el enfoque formativo inclusivo de evaluación que la Secretaría de Educación pública (SEP) ha propuesto para la Educación Básica en el país.

A partir del año 2011, en la política educativa en México se considera a la evaluación como "un proceso para aprender, que permita al docente tener un referente para la mejora de su práctica, que dé oportunidad a que los alumnos desarrollen sus capacidades, sobre todo aquellas en las que muestran más dificultades para alcanzar los aprendizajes esperados" (SEP, 2011, p. 108). Con el acuerdo 696 (SEP, 2013), se instituye la evaluación como una valoración del rendimiento de los alumnos que permite que logren los aprendizajes esperados. Se promueve la evaluación como un proceso de mejora y de toma de decisiones que redunden en una educación de calidad.

Los planteamientos anteriores permiten apreciar la pertinencia de estudiar las prácticas evaluativas en educación preescolar e identificar si se realizan con enfoque formativo inclusivo. Lipman (citado en Camilloni, Celman, Litwin y Palou, 1998), considera que las prácticas evaluativas tienen como criterio la representatividad (implicación del tema a tratar), la significación (importancia del tema), y la diferenciación cognitiva (proceso reflexivo); tomar en cuenta estos criterios implica una evaluación globalizada e integral de la tarea realizada. Desde este planteamiento se deduce que la educadora, desde el momento en que realiza el diseño de su intervención, debe prever cómo realizará la evaluación de los aprendizajes de sus alumnos y alumnas, de allí que la realización de prácticas evaluativas con un enfoque formativo inclusivo requiera que las educadoras identifiquen los aspectos del currículo que ya dominan los alumnos y cuáles se tienen que desarrollar, así como el tipo de estrategias que pueden favorecer que los alumnos logren aprendizajes que le sean útiles para desenvolverse en su contexto.

\footnotetext{
${ }^{1}$ En México se denomina educación preescolar a la que se ofrece a los niños de 3 a 5 años y actualmente forma parte de la educación básica, junto con la primaria, la secundaria y la preparatoria. Los niños que asisten a preescolar son considerados alumnos en tanto están escolarizados y reciben un reporte de evaluación.

${ }^{2}$ En México, a las docentes del nivel de preescolar habitualmente se les llama educadoras. A partir de este momento se utilizará esta terminología.
} 


\section{Justificación}

\subsection{La evaluación desde la mirada internacional y de los expertos.}

Como consecuencia de la competitividad que demanda la globalización, en la actualidad se considera a la educación como el eje central para abatir las desigualdades sociales; actualmente se requiere formar personas competentes, capaces de aprender a aprender, lo que implica que sigan generando aprendizajes después de terminar su formación profesional. Lo anterior ha dado lugar a trasformaciones en los lineamientos que rigen el funcionamiento de los diferentes sistemas educativos de varios países de América, tal es el caso de El Salvador, Perú y México, entre otros; por ello, han surgido diferentes reformas con el objeto de incrementar la calidad de los servicios educativos para alcanzar ese objetivo de formar ciudadanos competentes. La evaluación de los sistemas educativos, como señalan Rivas, Sobrino y Peralta (2005), va a permitir que se garantice la calidad de la enseñanza vinculada a la intensificación de los procesos de valoración de los alumnos, de los profesores, de los centros y del sistema en su conjunto.

De acuerdo con la experiencia de los autores, en muchos casos la evaluación es vista por los profesores como un trámite o una imposición. Desde esta mirada se corre el riesgo de evaluar únicamente por cumplir, sin darle su sentido pleno, lo cual además puede propiciar que la evaluación se centre en el alumno, sin tener en cuenta otros factores que pueden incidir en sus aprendizajes, por ejemplo el papel que juegan los demás agentes educativos (profesores, padres de familia e instituciones). Por ello, se considera conveniente promover la evaluación como herramienta de indagación, análisis y reflexión que lleve a que las maestras realicen una planificación y a la identificación de las estrategias que favorecen o impiden que los alumnos desarrollen los aprendizajes esperados.

Santos (1995) señala que se pueden encontrar profesores que no tienen una idea clara de la relevancia educativa de la evaluación, mientras otros no conocen cómo construir o diseñar una evaluación que considere los lineamientos exigidos por el sistema educativo. Cuando el profesor no tiene una idea clara de la función de la evaluación, corre el riesgo de limitarse a evaluar únicamente para asignar un juicio de valor a los resultados, y no tomarlos en cuenta como elementos que guíen la mejora de su intervención docente.

La educación inclusiva requiere de métodos de evaluación inclusivos, los cuales implican que la evaluación no debe centrarse solamente en los objetivos cognitivos de una educación de calidad, sino que debe tomar en cuenta todas sus dimensiones, que son: respeto de los derechos, equidad, eficacia, eficiencia, relevancia y pertinencia (Naciones Unidas, 2013).

En relación con la implementación de una evaluación inclusiva, Agut (2010) comenta que ésta debe centrarse en el proceso, con el objeto de tomar decisiones con respecto a la operacionalización de la enseñanza y del aprendizaje del alumno. El autor manifiesta que

la evaluación inclusiva debe contar con las siguientes características: ser un proceso abierto y flexible, que fomente la participación activa de todos los alumnos, que promueva la toma de conciencia del proceso de enseñanza-aprendizaje, que contemple niveles de participación, uso de diversos materiales, uso de diversos lenguajes, flexibilidad de tiempo para el desarrollo de la tarea y fijar indicadores de evolución del aprendizaje para los alumnos. (p. 44) 
Murillo y Duk (2012) refieren que desde el contexto de la evaluación inclusiva no es apropiado concebir una evaluación para clasificar o comparar, sino que su finalidad debe ser identificar las ayudas que se requieren para facilitar el proceso educativo. Es decir, que la evaluación se debe centrar en el proceso y considerar no sólo las capacidades del alumno, sino también los factores contextuales.

Conviene subrayar que la European Agency (s/f) definió siete niveles de indicadores de evaluación inclusiva, los indicadores se centran en la descripción del papel que juega cada uno de los actores implicados: alumnado, padres de familia, profesorado, centros educativos, equipos de evaluación multidisciplinares, políticas y legislación. Es decir que se plantea una visión sistémica de la evaluación que contempla la intervención de todos y cada uno de los agentes educativos.

Para promover un enfoque formativo inclusivo se deben analizar las prácticas evaluativas, las cuales son el punto nodal del proceso de evaluación, en ellas se pueden percibir las finalidades que tienen éstas para el docente: el qué, cómo, cuándo y dónde constituyen puntos de partida para la promoción de las prácticas con enfoque formativo e inclusivo. Como señalan Santiuste y Arranz (2008), la evaluación inclusiva se debe promover desde las políticas educativas, es crucial considerar las necesidades de todos los alumnos, además de tomar en cuenta sus finalidades. Así, las políticas educativas deben garantizar que la evaluación sea accesible para todos los estudiantes, todos los procedimientos deben estar ligados al currículo y por tanto deben promover el aprendizaje. La evaluación inclusiva debe entonces ser considerada como un instrumento eficaz para el desarrollo de las potencialidades y habilidades, evitar que se distorsione el propósito formativo con el uso de instrumentos cuantitativos, comunicar a los agentes educativos los procedimientos, evaluar e identificar buenas prácticas y evitar procedimientos de evaluación burocráticos.

La evaluación inclusiva busca promover que la valoración de los alumnos sea flexible y erradique etiquetas y que se trabaje en función de los aprendizajes de cada alumno. Al respecto, Coll y Orunbia (2002) señalan que este tipo de evaluación está al servicio de todos los alumnos, y que desde este enfoque se evalúa durante toda la jornada escolar. Estos mismos autores mencionan que se debe tomar en cuenta que la planeación y evaluación son procesos paralelos, remarcan que la evaluación debe encaminarse a establecer qué sabe y cómo lo sabe el alumno. Otra cuestión esencial en el enfoque inclusivo de evaluación es la devolución de los resultados de la misma, que debe hacerse de tal modo que promueva que tanto los alumnos como los docentes reflexionen y realicen un balance de los logros y dificultades.

\subsubsection{Perspectiva de la evaluación en la educación básica en México}

Con el fin de atender los requerimientos de la Organización para la Cooperación y Desarrollo Económico (OCDE), México ha solicitado la realización de diversas evaluaciones externas con el fin de verificar el funcionamiento del Sistema Educativo Nacional (SEN), y éstas han sido realizadas por la misma OCDE e internas por parte del Instituto Nacional para la Evaluación Educativa (INEE).

Es importante señalar que a partir de la Reforma Integral de la Educación Básica (RIEB) 2011, que tuvo por objeto la articulación de los niveles de educación preescolar, primaria y secundaria, se modificaron los programas para que fueran coherentes con el enfoque de competencias (SEP, 2004). Sin embargo, esta implementación del currículo por 
competencias no se realizó de manera simultánea, pues primero se inició en preescolar en el 2004, en secundaria en 2009 y en primaria en 2011.

Los cambios promovidos por la RIEB obligaron que se buscaran enfoques de evaluación acordes con el trabajo por competencias; en el Acuerdo 696 se estipularon las normas generales para la evaluación, acreditación, promoción y certificación en la educación básica (SEP, 2013). Este Acuerdo se fundamenta en la Ley General de Educación, específicamente en el Artículo 50. En el caso particular de la educación preescolar, en un documento llamado Guía para la Educadora 2011 (SEP, 2011), se especifica que la educadora es la responsable de llevar a la práctica el enfoque formativo e inclusivo de la evaluación de los aprendizajes. Sin embargo, en éste y otros documentos elaborados por la SEP no se describe cómo llevar a la práctica dicho enfoque.

\subsubsection{La Evaluación en educación preescolar en México}

La educación preescolar se ha evaluado mediante la Escala de Medición y Niveles de Logro (EXCALE), la cual se aplica a los niños de $3^{\circ}$ de preescolar con el objeto de verificar los conocimientos que tienen en las áreas de pensamiento matemático y de lenguaje y comunicación. La aplicación de la EXCALE de 2011 (INEE, 2014) permitió concluir que en el campo formativo de lenguaje y comunicación al menos el 94\% de las niñas y los niños alcanzó el nivel de logro básico. Este resultado es favorable, pues indica que al término del preescolar nueve de cada 10 alumnos mostraron un dominio básico o superior en relación con las competencias relacionadas con la lengua oral y escrita. De igual manera, en pensamiento matemático el $91 \%$ de los niños logró al menos el nivel básico. Los resultados anteriores muestran que las educadoras han realizado esfuerzos para que sus alumnos alcancen mejores niveles de logro en los campos evaluados, pero esta situación sigue ocasionando que le resten importancia a otros campos que quizá valga la pena fortalecer en el Jardín de Niños, por ejemplo el relacionado con el desarrollo personal social.

Las educadoras deberían ser las mediadoras de los aprendizajes de sus alumnos, percibiéndose como profesionales reflexivas con respecto a su práctica, promotoras de que el alumno sea partícipe de su formación (que autorregule su aprendizaje), promotoras asimismo de que los padres de familia se involucren en la formación de sus hijos $\mathrm{y}$, finalmente, promotoras de procesos de educación inclusiva, todo lo anterior mediante el trabajo colaborativo y sistemático, basado en los resultados de la evaluación. Vale la pena decir que en el nivel de preescolar en México se han dado casos de inclusión que han sido más favorables que en otros niveles de la educación básica (SEP, 2010). Aun así, resulta complejo para las educadoras entender el concepto de inclusión, el cual va más allá de atender al alumnado que tenga una discapacidad, implica que se busque que todos los alumnos aprendan, respetando sus ritmos y estilos de aprendizaje. Es por ello que resulta de vital importancia la realización de una investigación que dé cuenta del tipo de prácticas evaluativas que realizan las educadoras.

\subsection{Fundamentación}

En México, a pesar de que en el nivel de preescolar se inició en la década de los 60 con la escuela de párvulos, se declaró obligatorio, por decreto presidencial, hasta el año 2003; a partir de ese año se incluyó dentro de la educación básica. Su obligatoriedad fue 
paulatina, debido a las dificultades para alcanzar la cobertura del nivel ${ }^{3}$. A pesar de esto, de acuerdo con la experiencia de los investigadores, los padres de familia siguen dando más valor a que sus hijos cursen sólo los dos últimos grados de los tres que conforman el nivel $^{4}$, lo que impide que haya continuidad en la adquisición de los aprendizajes de las alumnas y alumnos; este hecho representa un reto para las educadoras porque, dadas las exigencias de los programas de estudio, tienen que nivelar los aprendizajes de los niños que ingresan tardíamente con los del resto de sus compañeros.

Se debe mencionar que el horario de servicio del preescolar en México es de 9 a $12^{5}$ horas, es decir que se atiende a los niños tres horas. En este nivel se ha buscado ofertar materias de música y educación física y, a partir de su obligatoriedad, una lengua adicional (en este caso de inglés) únicamente para los alumnos de tercer grado. Las autoridades han implementado estos servicios en los Jardines de Niños, aunque aún no se ha logrado dar cobertura a todas las escuelas (en especial el de inglés), debido a la falta de plazas y docentes; así, se ha optado por capacitar a las educadoras para que ellas impartan esta materia ${ }^{6}$.

En concordancia con la declaración de obligatoriedad del nivel surgió el Programa de Educación Preescolar 2004 (PEP) (SEP, 2004), en el cual se comenzó a enfatizar la función formativa de la evaluación. En este documento se daba importancia a la realización del diagnóstico como punto de partida para detectar los aprendizajes previos, las dificultades y lo que podrían llegar a hacer los alumnos, y a partir de ello diseñar las estrategias de intervención para el logro de las competencias. La evaluación, en el programa mencionado, se definió como un proceso de valoración en el que se emite un juicio basado en la información que la educadora recoge a lo largo del ciclo escolar, tomando como referencia las observaciones del trabajo diario ${ }^{7}$. La implementación del PEP 2004 requería que las educadoras fueran profesionales capaces de tomar decisiones complejas de manera reflexiva, para desarrollar su práctica sobre la base de conocimientos sólidos acerca del qué y cómo enseñar.

Por consiguiente, a partir de la obligatoriedad del nivel de preescolar las educadoras han tenido que presentar resultados de los aprendizajes de los alumnos, lo que ha propiciado que las prácticas educativas, y en especial las evaluativas, tengan ajustes drásticos. Mientras que en los programas anteriores interesaba en especial el desarrollo afectivo del niño, en últimas fechas interesa el desarrollo cognitivo. Al respecto, Rivera y Guerra (2005) manifiestan que desde la obligatoriedad del preescolar, en la política educativa existe interés por impulsar un nuevo esquema, a tono con las tendencias institucionales de la reforma educativa. Uno de los principales retos que se ha impuesto el nivel es que

\footnotetext{
${ }^{3}$ La matrícula, de acuerdo con la Secretaria de Educación Pública para el ciclo escolar 2013-2014, fue de 107,954, de los cuales fueron 54,480 hombres y 53,474 mujeres.

${ }^{4}$ De acuerdo con la estadística reportada por la Secretaria de Educación Pública para el ciclo 2014-2015 el porcentaje de niños inscritos de 3 años fue del $42.2 \%$, de 4 años de $89 \%$, de 5 años del $84.3 \%$.

${ }^{5}$ Recientemente, el secretario de Educación anunció que a partir del ciclo escolar 2016-2017 el horario aumentará a cuatro horas.

${ }^{6}$ Esta información fue proporcionada por la asesora técnica en un curso de capacitación para personal directivo de jardines de niños particulares.

${ }^{7}$ La educadora registraba en el Diario de la educadora las observaciones de lo que acontecía en la jornada de trabajo con los niños, con lo cual se buscaba que reflexionara sobre la eficacia de las estrategias empleadas y realizara ajustes a su intervención docente.
} 
las educadoras tengan una mayor autonomía para la toma de decisiones, la cual redunde en una intervención docente creativa y reflexiva.

Con objeto de conocer la opinión de las educadoras respecto a la implementación del PEP 2004, la SEP realizó jornadas de trabajo en las que ellas manifestaron la necesidad de fomentar el análisis y la reflexión sobre sus prácticas pedagógicas. Además, mencionaron que hay factores externos que influyen en las situaciones problemáticas de los niños que la escuela se ve limitada a resolver. Algunos de los principales problemas que refirieron fueron: inconsistencia entre lo que se planea, se realiza y se evalúa, falta de sistematización del trabajo y del uso del tiempo en la intervención docente y no retomar los saberes previos de los niños; en lo que respecta a la evaluación, plantearon que los materiales son poco pertinentes, adecuados y relevantes, además que aplican la evaluación de acuerdo con sus propias interpretaciones con respecto a lo que debería ser dicho proceso, por ello puede suceder que los resultados que se obtengan no sean reales (SEP, 2004). A pesar de que los resultados de dichas jornadas tenían que ser un referente para la implementación de un nuevo programa (SEP, 2011), aparentemente persistieron las dificultades experimentadas con el programa anterior, sobre todo la ausencia de reflexión sobre la práctica docente y sobre la evaluación de los alumnos.

En el Programa de estudios 2011 se estipuló que el enfoque formativo inclusivo en el proceso de evaluación no sólo se centra en el alumno, sino también en el docente, en los padres de familia y en el centro de trabajo. Se trata de promover una cultura de evaluación. Además, se hace referencia a que se realice la autoevaluación, coevaluación y heteroevaluación con el fin de promover la autorregulación del alumno en su proceso de aprendizaje, con lo que se promueve el desarrollo de la metacognición ${ }^{8}$.

Asimismo, atendiendo a las mejores prácticas en materia de evaluación de aprendizajes, la SEP determinó implementar un modelo de evaluación que considera lo cualitativo y lo cuantitativo, donde se describen los logros y dificultades de los alumnos; esta evaluación se apoya fuertemente en la observación y en el registro de información por parte de la educadora durante el desarrollo de las actividades (SEP, 2013, p. 5); lo anterior implica que:

- La educadora planifique actividades para que los alumnos estudien y aprendan;

- los alumnos se den cuenta de lo que han aprendido y de lo que están por aprender;

- las educadoras tomen en cuenta los procesos de aprendizaje, no sólo los resultados;

- las educadoras consideren las necesidades específicas de los alumnos y de los contextos en los que se desarrollan;

- las educadoras obtengan la información sobre el desempeño de los alumnos de distintas fuentes no sólo de las pruebas;

\footnotetext{
${ }^{8}$ La actividad metacognitiva supone la capacidad de planificar qué estrategias se han de utilizar en cada
} situación, aplicarlas y evaluar su funcionalidad y a partir de esto replantearlas. 
- en la institución se fortalezca la colaboración entre educadoras, alumnos y padres de familia o tutores;

- en la institución, los profesores y autoridades educativas actúen oportunamente para evitar el rezago o la deserción escolar.

Por lo tanto, se enfatiza que la evaluación debe ser un proceso sistemático, organizado, que la educadora debe considerar a la hora de diseñar la planeación, con el propósito de definir los aprendizajes que pretende que el alumno alcance al término de un periodo escolar. Es un proceso que implica la toma de decisiones, lo que pone en juego la capacidad de análisis y reflexión de quien lo realiza, con el fin de emitir juicios y replantear acciones para que todos los alumnos tengan oportunidades de alcanzar los aprendizajes esperados.

En este tenor, Antúnez y Aranguren (2004) mencionan que la reflexión es importante para que el docente realice ajustes a su intervención. Desde esta postura, señalan que la evaluación se concibe como la verificación de la información asimilada y como un medio de acreditar conocimientos construidos por el alumno. Sin embargo, la reflexión sobre los resultados es una tarea que la mayoría de las educadoras aún mantienen pendiente, debido a múltiples factores, en especial por la saturación de tareas administrativas.

\section{Método}

Objetivo general: caracterizar las prácticas evaluativas con enfoque formativo inclusivo de educadoras de educación preescolar

Objetivos específicos:

- Conocer cómo han interpretado las educadoras el enfoque formativo, establecido en el programa de estudios 2011. Guía para la educadora.

- Identificar qué tipo de prácticas realizan las educadoras de acuerdo con las interpretaciones que han construido del programa de estudios 2011.

Diseño

El presente estudio es de tipo cualitativo, sigue los lineamientos de la investigaciónacción participativa. Esta modalidad de investigación promueve el trabajo colaborativo entre un experto y los participantes del estudio. La investigación-acción, desde el punto de vista de Car y Kemiss (1988, en Latorre, 2003, p. 31) tiene como objetivo "la comprensión, la transformación y la toma de conciencia, en la cual el rol del investigador es socrático, pues propicia la participación y la autorreflexión”.

\section{Contexto}

El estudio se realizó en un Jardín de Niños público ubicado en una zona conurbada de la ciudad de San Luis Potosí. El Jardín es de organización completa, cuenta con tres grupos de cada grado (atendidos por sus respectivas educadoras). En promedio, en cada grupo 
hay entre veinte y veinticinco alumnos. En el plantel se imparten las clases de música, inglés y educación física. Se cuenta con el servicio itinerante del personal de USAER ${ }^{9}$.

\section{Participantes}

Participaron tres educadoras, dos de segundo de preescolar y una de tercero. El trabajo de campo se realizó en el ciclo escolar 2014-2015. Para la selección de las participantes sólo se consideró su disposición para participar voluntariamente en el estudio. Las tres educadoras participantes cuentan con una antigüedad de 28, 30 y 31 años de servicio, respectivamente.

\section{Instrumentos}

Para realizar la indagación de las prácticas evaluativas de las educadoras, se realizaron observaciones de aula (Postic y De Ketelle, 1992), además se aplicó una entrevista semiestructurada (Taylor y Bodgan, 1994). Finalmente, se revisaron los planes de trabajo, los instrumentos de evaluación y los portafolios de algunos niños. Para la presentación y tratamiento de los datos se asignaron nombres ficticios para resguardar la identidad de las educadoras participantes.

\section{Técnicas de análisis de datos}

Para el análisis se codificaron y categorizaron los datos de acuerdo con Latorre (2003), que implica asignar códigos, que son las etiquetas que vinculan los fragmentos de texto o unidades de análisis a las categorías. Codificar significa fragmentar en unidades de significado o de análisis, al que se le otorga significado propio. Se establecieron las siguientes categorías de análisis: evaluación formativa, evaluación inclusiva y prácticas evaluativas. Se consideró más conveniente la realización del análisis de los datos como se ha descrito, dada la naturaleza del objeto de estudio, ya que dicho procedimiento posibilita su análisis e interpretación.

\section{Resultados}

Los resultados se han organizado de acuerdo con las preguntas de la investigación, tratando de evidenciar con los datos empíricos los hallazgos en relación con las prácticas evaluativas de las educadoras.

\section{1. ¿Cómo han interpretado las educadoras el enfoque formativo, establecido en el Programa de Estudios 2011. Guía para la Educadora?}

Las educadoras manifiestan estar interesadas en seguir los lineamientos proporcionados en el documento mencionado, buscan capacitarse para estar actualizadas, procuran mejorar su labor ya sea mediante el trabajo colegiado con sus compañeras, con su directora o con profesionales externos (como el Apoyo Técnico Pedagógico o con las USAER). La educadora Mayela comenta: "hemos estado revisando textos diferentes de lo

\footnotetext{
${ }^{9}$ Unidades de Servicio de Apoyo a la Educación Regular. Se trata de equipos de educación especial que apoyan a la integración de alumnos con necesidades educativas especiales a las escuelas regulares, conformados por cinco especialistas: director, psicólogo, maestro de comunicación, trabajador social y maestro (s) de apoyo (pueden ser más de uno). Trabajan de manera itinerante en cinco escuelas, con excepción de los maestros de apoyo, que trabajan en una.
} 
que es la evaluación, de cómo hacer el diagnóstico, qué evaluar, qué referencias tomar, qué es lo que nos falta” (EMM, 2014).

Las entrevistadas coincidieron en que han tenido dificultades para la implementación de los dos últimos programas (2004 y 2011), porque les llevó tiempo comprender cómo realizar su intervención docente bajo el enfoque por competencias, especialmente entre las educadoras que tienen más años de servicio (más de 30 años) y que han tenido oportunidad de implementar varios programas. Como indica la educadora, América: "ha sido una situación muy difícil para mí, ya tantos años de trabajo y cambie y cambie, realmente ha sido difícil para mí "(EEA- Entrevista Educadora America, 2014, p. 10). En contraparte, las educadoras que sólo han trabajado con estos programas (2004 y 2011) tuvieron menos dificultades en su aplicación.

Las educadoras tienen claro el sustento teórico de la evaluación formativa, dado que mencionan que es una evaluación centrada en conocer tanto logros como dificultades de los alumnos, como lo manifestó la educadora Ximena ${ }^{10}$ : "ahora vemos los logros y dificultades en los niños, la evaluación es un proceso que busca ver los avances de los niños" (EEX, 2014). Mayela, al respecto, menciona "la evaluación son los alcances que tiene el niño a partir de los aprendizajes que queremos lograr" (EEM- Entrevista Educadora Mayela., 2014).

Otra función que las educadoras perciben de la evaluación es que sirve para mejorar su intervención docente, pues se realiza una valoración de los aprendizajes que sirve como referente para la planeación de su intervención. Tal como lo expresa América: "a partir de ahí voy a planear en la siguiente oportunidad que tengo de obtener mejores logros" (EEA, 2014, p. 10). Sin embargo, en la práctica consideran que la evaluación se realiza en cortes de tiempo y no continuamente; en cada actividad de inicio que ponen en práctica pretenden verificar los aprendizajes previos de los alumnos, en las de desarrollo los niños ponen en juego sus habilidades y aptitudes y en las de cierre verifican qué nuevos aprendizajes adquirieron con la realización de las actividades. Esto se puede apreciar de manera más detallada en el tabla 1, en el que se plantean las evaluaciones realizadas por Mayela.

Las educadoras reconocen que para realizar una buena evaluación se requiere del apoyo de los padres de familia, tal y como se plantea en el programa 2011; al respecto, Ximena expresa: "para que se logre una buena evaluación se debe de contar con la participación de los padres de familia" (EEX, 2014). Este hecho evidencia que la evaluación involucra factores que van más allá del aula, por lo que la visión del proceso debería ser más global, no sólo como lo estipula el programa.

Otro hallazgo es que las educadoras reconocen que la evaluación en educación preescolar siempre ha sido cualitativa, como lo manifiesta América: "la evaluación siempre ha sido cualitativa, preescolar no cuantifica, se expresa por medio de palabras" (EEA, 2014, p. 10). Al ser la evaluación cualitativa, las educadoras describen los logros y dificultades de sus alumnos y los apoyos que requieren.

La evidencia empírica presentada refiere que las educadoras han intentado poner en práctica la evaluación formativa, ya que realizan una valoración de los aprendizajes de

${ }^{10}$ América, Ximena y Máyela son educadoras. A partir de este momento se omitirá su profesión con el fin de agilizar la lectura. 
sus alumnos, aprecian las dificultades y reestructuran estrategias para promover que todos alcancen los aprendizajes esperados, es aquí en donde entra la vertiente inclusiva de la evaluación; además, Mayela y Ximena se centran en los niños que presentan mayores dificultades para acceder a los aprendizajes, asignándoles actividades que apoyen su seguridad y remarcando los logros que tienen. Sin embargo, falta continuar trabajando en la diversificación de instrumentos de evaluación acordes a las necesidades de los alumnos, esto atendiendo también al diseño universal de aprendizaje, el que estipula que se debe diversificar la enseñanza para brindar la oportunidad de que todos los alumnos desarrollen sus habilidades. 
Tabla 1. Ejemplo de Actividades de inicio, desarrollo y cierre que realiza Mayela

\begin{tabular}{|c|c|c|}
\hline ACT. DE INICIO & ACT. DE DESARROLLO & ACT. DE CIERRE \\
\hline $\begin{array}{l}\text { Maestra: El día de hoy vamos } \\
\text { a hacer otra actividad } \\
\text { Niños: siii... jugar con los } \\
\text { títeres. } \\
\text { Maestra: Ah bueno, además de } \\
\text { jugar con los títeres... Hoy } \\
\text { me encontré con un problema } \\
\text { muy grande me encontré con } \\
\text { estas láminas. } \\
\text { Maestra: si hay animalitos. } \\
\text { Entonces se trata de que } \\
\text { ustedes me digan cuántas } \\
\text { fichas o cuántas semillitas o } \\
\text { bolitas necesitarían para, como } \\
\text { por ejemplo, llegar a donde } \\
\text { está la mosca ¿cuántos creen? }\end{array}$ & $\begin{array}{l}\text { Maestra: a ver fíjense bien, } \\
\text { ojitos para acá. Brazos } \\
\text { cruzados, bien prensados, } \\
\text { ojitos en el pizarrón. Fíjense } \\
\text { bien cómo vamos a trabajar } \\
\text { todos ahorita. Fíjense bien, a } \\
\text { unos les va a tocar poner las } \\
\text { fichas, van a contar cuántas } \\
\text { fichas van a poner, otros van a } \\
\text { tener la hoja de registro donde } \\
\text { van a estar apuntando quiénes } \\
\text { son los equipos que van a } \\
\text { ganhar }\end{array}$ & $\begin{array}{l}\text { La educadora por lo regular } \\
\text { realiza preguntas a los niños } \\
\text { en asamblea, como por } \\
\text { ejemplo: ¿Qué les gustó? ¿Qué } \\
\text { trabajaron durante el día? }\end{array}$ \\
\hline
\end{tabular}

Fuente: Elaboración propia a partir de (OC, 2014).

\section{2. ¿Qué tipo de prácticas realizan las educadoras, de acuerdo con las interpretaciones que han construido del plan de estudios?}

Para efectos de este trabajo se considera que las prácticas evaluativas se refieren a cómo instrumentalizan las educadoras el proceso de evaluación, incluye las concepciones que tienen respecto a lo que es evaluar, a los instrumentos que utilizan, al tipo de evidencias que presentan, la forma de reportar los resultados, el uso que le dan a dichos resultados, el papel que tienen tanto el alumno como la educadora y las modalidades de evaluación, entre otros.

Uno de los hallazgos de esta investigación es la inconsistencia en la sistematización del proceso de evaluación, que puede dar como resultado que no exista un diagnóstico ${ }^{11}$ fidedigno, lo anterior se ha podido observar en la realización del trabajo del campo y en la revisión de documentos (por ejemplo la revisión de planes de clase), en los instrumentos de evaluación, y en las evidencias de aprendizajes. "El no llevar una sistematización es cuestión de tiempo, si nos dieran tiempo lo hiciéramos más a conciencia” (EMX- Entrevista Educadora Ximena, 2014, p. 10).

Durante la observación se pudo identificar que en el trabajo de las educadoras se muestran inconsistencias entre lo que se planea y la forma en que se evalúa. Se puede verificar en la planeación un inicio, desarrollo y cierre de las actividades; las educadoras consideran que planear y evaluar son dos procesos separados, en especial Ximena, quien evidencia una gran preocupación por el diseño de instrumentos para evaluar los aprendizajes de los niños. En este sentido, las educadoras comentan tener claro lo que es la evaluación, aunque aceptan su complejidad.

En cuanto al uso de los instrumentos de evaluación, América emplea el Diario de la educadora, en el cual hace una narración de las actividades que realizó durante la jornada, pero su análisis muestra falta de reflexión sobre su práctica.

11 Para efectos de este estudio se entiende como diagnóstico al proceso que permite visualizar los aprendizajes previos de los alumnos, así como las áreas de oportunidad que se tienen que trabajar en ellos, además del tipo de estrategias que permiten fortalecer sus aprendizajes. 
Por su parte, Ximena hace uso de una escala estimativa y de la rúbrica; divide cada uno de los expedientes de los niños por campos, y en cada campo maneja como evidencias un dibujo o trabajo que sustenta el desarrollo de los niños; además, desglosa los indicadores que se espera que los niños alcancen o están en proceso de alcanzar. Los campos formativos son: exploración y conocimiento del mundo, desarrollo físico y salud, lenguaje y comunicación y pensamiento matemático; en cada uno de los campos incluye las evidencias de los aprendizajes de los alumnos, por ejemplo trabajos que evidencien el nivel de conceptualización con respecto a la escritura. Al reverso de las evidencias describe el aprendizaje esperado, competencia, campo formativo, aspecto y la fecha. También realiza una descripción del desempeño del niño en la actividad (rúbrica). En el campo formativo de pensamiento matemático utiliza una escala estimativa, coloca el aspecto a evaluar, los niveles de logro (lo logra, lo logra con ayuda, no lo logra) y lista los aspectos a evaluar. En el apartado de desarrollo personal y social y expresión y apreciación artística también hace uso de una escala estimativa. Se pudo apreciar que hay más evidencias e instrumentos en los campos formativos de pensamiento matemático y de lenguaje y comunicación.

Por lo que refiere a Mayela, comentó durante la entrevista que, con respecto al uso de instrumentos de evaluación, no utiliza actualmente el diario de la educadora, lo utilizó por mucho tiempo porque desde su punto de vista es útil, pues si no se escribe no se recuerda; sin embargo, es tanto el trabajo administrativo que empezó a utilizar otros instrumentos, como la rúbrica o la lista de cotejo, aunque ésta le ha sido difícil de implementar por la complejidad que representa la definición de indicadores que le permitan contemplar en qué medida los alumnos adquirieron el aprendizaje esperado.

Ximena busca que los alumnos participen activamente durante las actividades, realiza constantemente cuestionamientos para incitarlos a que piensen acerca de las posibles respuestas, y cuando no responden correctamente realiza la corrección. Por ejemplo, en una de las observaciones la educadora preguntó a los alumnos cómo escribir algunas palabras, entre ellas la palabra "hombro". La mayoría de los niños escribió "ombro", la docente siguió cuestionando, pero los niños insistieron. Ella hizo la corrección y escribió correctamente la palabra a un costado de donde los niños la habían escrito, y les explicó que se escribía con "h" y que la letra no sonaba.

En cuanto a América, se observó que hace cuestionamientos del tipo ¿qué es? ¿A qué huele?, por ejemplo cuando está revisando el tema de los sentidos, pero en ocasiones no queda claro el propósito de la actividad, con esto corre el riesgo de no explorar adecuadamente los conocimientos de los alumnos. Por ejemplo, como se ilustra en la transcripción de la clase que aparece más adelante, la educadora pregunta a uno de sus alumnos a qué huele, y él contesta a áspero, pero la educadora no indaga sobre esta respuesta. Se observó que América promueve más el trabajo individual en comparación con el grupal o de pequeños grupos. La educadora utiliza constantemente dibujos que hacen referencia al tema que está revisando y pide a los niños que los iluminen o decoren con algún material, después ella pide a cada niño que le explique lo que hizo, para conservarlo como evidencia de aprendizaje (tabla 2 ).

Por su parte, las prácticas evaluativas de Mayela consisten en dar la consigna, verificar con un ejemplo que la consigna haya sido entendida por los niños, y en todo momento promover la participación de todos los alumnos, además de promover el trabajo en grupo y en pequeños grupos. Se observó que la educadora propicia la toma de decisiones en 
consenso a la hora de trabajar en equipos. Otra característica de su práctica es que permanece atenta durante toda la actividad para observar el desempeño de todos sus alumnos.

Tabla 2. Ejemplo de dinámica de clase de las educadoras: América, Mayela y Ximena

\begin{tabular}{|c|c|c|}
\hline AMERICA & MAYELA & XIMENA \\
\hline $\begin{array}{l}\text { M: toma un frasco de resistol } \\
\text { y comienza a pasárselos a los } \\
\text { niños para que lo huelan. } \\
\text { M: ¿a qué huele? } \\
\text { Niño: xxx } \\
\text { M: ¿a qué? } \\
\text { Niño: áspero. } \\
\text { M: ¿áspero?, ¿áspero? } \\
\text { M: huele a áspero [la docente } \\
\text { se ríe] }\end{array}$ & $\begin{array}{l}\text { M ....chicos atención fíjense } \\
\text { bien en dónde voy a poner la } \\
\text { ficha. Chicos, ¿cuántas fichas } \\
\text { se necesitarán para llegar a la } \\
\text { araña barrigona? } \\
\text { N: siete } \\
\text { M: ¿cuántos crees? } \\
\text { N: siete, maestra, para llegar } \\
\text { aquí } \\
\text { N: ocho, ocho maestra } \\
\text { M: ¿cuántas fichas necesitaron } \\
\text { desde la casita hasta llegar a la } \\
\text { araña. Cuántas fichas } \\
\text { necesitaron para llegar hasta } \\
\text { la araña? } \\
\text { (...) M: seis. Fuera las fichas } \\
\text { porque vamos a ver ahora } \\
\text { quien ganó. Ya apunté cuántos } \\
\text { dice cada quien. El equipo Uni } \\
\text { sumi dice que siete, el equipo } \\
\text { Río dos dice que ocho, el } \\
\text { equipo Rayo Mac Queen dice } \\
\text { que ocho y el equipo Max } \\
\text { steel dice que seis. Vamos a } \\
\text { contarlas a ver ayúdenme. } \\
\text { N: uno, dos, tres, cuatro, } \\
\text { cinco, seis, siete, ocho. } \\
\text { M: ¿quién gano? ¿Quién dijo } \\
\text { que ocho? } \\
\text { N: yo }\end{array}$ & $\begin{array}{l}\text { M: ¿y quién puede hacerme el } \\
\text { cinco? } \\
\text { Algunos niños. ¡yo!, ¡yo! } \\
\text { M: ¡aquí Fer lo va a hacer! } \\
\text { M: aquí, cinco ¡Grande! } \\
\text { M: ¡a ver ¡Quién más me } \\
\text { puede pasar a escribir el } \\
\text { número cinco? } \\
\text { M: le da el gis a David para } \\
\text { que escriba en el pizarrón. } \\
\text { M: sí, Fer, pero tienes que } \\
\text { hacerle un palito. ¡Fíjate bien! } \\
\text { M: una rayita y su } \\
\text { pancita....que no se les olvide } \\
\text { M: ¡muy bien Fer!, ¡muy bien } \\
\text { David!... Pero hay que hacerlo } \\
\text { más grandecito } \\
\text { M: ¡a ver ¡ ¡Héctor!, ¡Héctor! } \\
\text { M: me gusta, Héctor, porque } \\
\text { ya empezamos a trabajar } \\
\text { diferente. } \\
\text { M: ¿cuál está mejor? } \\
\text { Algunos niños: ¡el de Héctor! } \\
\text { M: hay que procurar hacerlo } \\
\text { como Héctor. }\end{array}$ \\
\hline
\end{tabular}

Fuente: Elaboración propia.

En otro asunto, en la presente investigación se encontró que hay algunos factores que inciden en las prácticas evaluativas, como la gestión pedagógica e institucional, la autonomía profesional, las percepciones de las educadoras, y los factores contextuales.

En cuanto a la gestión pedagógica, las educadoras encuentran barreras en la manera de gestionar o dirigir su labor docente, dados los requerimientos que les imponen las autoridades educativas. Lo anterior puede dar lugar a que las educadoras se vean limitadas para ejercer su autonomía profesional a nivel áulico. Al respecto, Ximena señala: "Uno de los retos que hemos enfrentado es el uso de instrumentos que vienen en el programa... el reto es que la asesora técnica diga esto le falta a tu portafolio... este es uno de los principales problemas que nos enfrentamos... trato de ponerle todo lo que nos piden“ (EEX, 2014).

En consecuencia, que las educadoras se vean limitadas para gestionar los aprendizajes al interior del aula propicia que vean como un trámite la elaboración de la planeación y, en algunos casos, diseñen una planeación para satisfacer los requerimientos institucionales o de las autoridades educativas, y otra planeación en la que plasman los aspectos que se deben trabajar con los niños de acuerdo a lo que observan en la jornada de trabajo. 
Se debe consignar que las educadoras acatan indicaciones de las autoridades educativas sin cuestionarlas, pareciera que sólo se limitan a seguir órdenes, cuando en realidad ellas son las que conocen a sus alumnos y saben cómo aprenden, qué necesidades de aprendizaje tienen y los apoyos que requieren; en suma, son las expertas. Esta situación puede ocasionar que la autonomía profesional de las educadoras se vea disminuida, pues cabe señalar que el aula es el único espacio en el que ellas tienen la posibilidad de tomar decisiones respecto a su intervención, basándose en su experiencia docente y su profesionalización.

En cuanto a los factores contextuales que interfieren con las prácticas evaluativas, las educadoras refieren que los padres de familia cuentan con bajos recursos económicos, constituyen familias desintegradas y que en los alrededores de la comunidad hay pandillerismo y violencia; estos factores se reflejan en el aula, sobre todo en la falta de apoyo de los padres de familia con respecto al desarrollo de los aprendizajes de sus alumnos.

\section{Discusión y conclusiones}

En algunas investigaciones (Gómez y Seda 2008; Martínez y Rochera, 2010), se afirma la necesidad de realizar estudios que evidencien la falta de capacitación e información docente oportuna, así como la dotación de recursos y materiales para la puesta en marcha de las reformas que se plantean a nivel gubernamental, no sólo en el nivel de preescolar, sino en el resto de los niveles de la educación básica (primaria y secundaria).

Es de vital importancia que en el transcurso de las investigaciones se dé voz a las docentes, sobre todo en un momento histórico en el que su figura ante la sociedad se ha visto devaluada, señalada, asociada con personas carentes de interés e iniciativa, que pareciera que no tienen gusto por su profesión; por ello es necesario mostrar facetas más positivas, pues sin duda hay educadoras comprometidas con su labor, que son profesionales que se capacitan, que intentan comprender lo que se requiere de ellas y realizan su práctica educativa con los escasos recursos con los que cuentan, enfocadas en lograr que todos sus alumnos desarrollen aprendizajes significativos.

En cuanto a las prácticas evaluativas, queda claro que las educadoras participantes del estudio utilizan algunos instrumentos de evaluación, en particular la rúbrica. En cuanto al diario de la educadora, consideran que es muy útil para el análisis de su práctica, sin embargo requiere una considerable inversión de tiempo. Lo anterior da pauta para considerar que si se promueve en las educadoras la reflexión como una estrategia que contribuya al análisis de sus prácticas, podrán tomar decisiones de manera fundamentada, donde valoren lo que funcionó y lo que tienen que modificar y que esta toma de decisiones redunde en los aprendizajes de sus alumnos, con el fin que todos tengan oportunidades para aprender. Es necesario entonces promover una evaluación formativa e inclusiva, que se centre en el proceso de cada alumno en individual y reconozca sus estilos y ritmos de aprendizaje.

Así pues, para promover una evaluación inclusiva se requiere sistematización de lo que el docente planea y evalúa; es decir, se deben evaluar los aprendizajes que se contemplaron en la planeación, por tanto la evaluación debe evidenciar los logros, dificultades y acontecimientos importantes, tanto relacionados con el desempeño del docente como de los alumnos. Como refiere Santos (1995), la evaluación no es un proceso centrado en el 
alumno, sino que debe propiciar la mejora de la intervención docente y del colectivo escolar.

Otro aspecto que puede contribuir a realizar mejoras en las prácticas evaluativas es el trabajo colaborativo, el cual podría contribuir a que las educadoras superen la visión de que la evaluación es un proceso complicado, tedioso, y un mero trámite. Hace falta continuar trabajando con las educadoras desde un enfoque comprensivo, donde se reconozcan los referentes epistemológicos desde los cuales realizan sus prácticas evaluativas

Durante el trabajo de campo se constató de voz de una de las educadoras la preocupación por dar cumplimiento a las demandas que ha tenido con respecto a la evaluación. La profesora expresa que está abrumada porque no ha logrado evidenciar en la evaluación los productos relacionados con los contenidos actitudinales, procedimentales y conceptuales, esto no le queda claro y está buscando información al respecto. La misma educadora describió su inquietud porque los niños de tercero de preescolar a su cargo no logran realizar el trazo del número dos. Otra educadora refiere que tiene dificultades para evidenciar el grado en el que los niños se encuentran en relación con la comprensión lectora; a pesar de que ha indagado, no ha encontrado un instrumento que le permita dar cuenta de este aspecto. En definitiva, lo anterior hace pensar que las educadoras han tenido que lidiar con las interpretaciones que las autoridades han realizado acerca de cómo realizar el proceso de evaluación, no obstante estas interpretaciones se centran en la entrega de resultados, más que en el proceso .

Desde la obligatoriedad del Preescolar, el nivel sufre presiones por el logro por parte de los alumnos de la lectura y la escritura y de nociones básicas del pensamiento matemático, por ello en este contexto se percibe preocupación por aspectos que parecieran formar parte de otro nivel de educación básica. Esta situación evita que se dé un enfoque formativo inclusivo, al excluir a los niños que presentan dificultades para adquirir las competencias mencionadas.

Por lo anterior, es evidente que las educadoras requieren de acompañamiento $\mathrm{y}$, simultáneamente, de autonomía para realizar sus prácticas; asimismo, requieren de espacios para realizar el trabajo colaborativo. Hace falta, por tanto, continuar con estudios que promuevan el acompañamiento de la labor docente como apoyo para la mejora de sus prácticas evaluativas, en las cuales se considere el enfoque formativo inclusivo.

En la actualidad, los docentes de educación básica tienen que someterse a una evaluación ${ }^{12}$ periódica (cada cuatro años, de acuerdo con el Instituto Nacional de Evaluación de la Educación) para garantizar su permanencia dentro del sistema educativo, lo cual es contradictorio con lo que solicita que realicen con sus alumnos, ya que dicha valoración es sumativa, no se le da seguimiento, no se centra en las áreas de oportunidad sino sólo en los resultados; estos últimos, como se sabe, pueden ser poco válidos, pues solo toman en cuenta aspectos aislados de la labor docente, lo que deja

12 Al respecto de la evaluación docente, autores como Díaz (2016) expuso que hay serias fallas en la instrumentalización de dicha evaluación que la hace injusta y carente de ética, pues con ésta se define si el docente es apto o no para la docencia. Además, hubo serios problemas de diseño del instrumento utilizado. 
entrever que las autoridades educativas plantean esta evaluación más con fines de control que de elevar la calidad de la educación.

En el sistema educativo mexicano se propuso en el Plan de Educación Básica la implementación de un enfoque formativo, y en el Plan de Educación Preescolar, además de ser formativo debía ser inclusivo; en el presente trabajo se encontró que las educadoras desconocen la terminología relacionada con el enfoque de evaluación, refieren el procedimiento que describe el programa sobre, sin embargo aún tienen incertidumbre respecto a los requerimientos relacionados con el diseño de instrumentos y de una evaluación diagnóstica. Se encontró también que las educadoras perciben que las autoridades educativas aminoran y descalifican sus esfuerzos por tratar de cumplir con los requerimientos, lo cual ocasiona que realicen prácticas de simulación para tratar de cumplir las demandas, hallazgo que se ha constatado en investigaciones con otros docentes, como la de García y Romero (2016).

En conclusión, se espera que los resultados de esta investigación inviten a la reflexión a las autoridades educativas y a la de las propias educadoras para que ambas partes actúen en consonancia y cambien el esquema de entrega de resultados o rendición de cuentas por el análisis de los resultados de evaluación o metaevaluacion, que promuevan una toma de decisiones consensuada que permita que todos los alumnos avancen, estableciendo prioridades para el trabajo docente con cada uno de ellos; lo anterior permitiría que se cambiara la visión de que el grupo tienen que ir al mismo ritmo de aprendizaje, pues cada niño es diferente y por lo tanto tiene diferentes intereses, habilidades y capacidades.

Es importante señalar que dado el método utilizado, no se pueden generalizar los resultados de esta investigación, por lo que se considera necesario realizar más estudios que aborden temas relacionados con las vivencias de los alumnos de la evaluación en educación preescolar, de lo que piensan los padres al respecto y donde se involucre a las autoridades educativas, dado que el presente estudio sólo se centró en las educadoras. También sería pertinente realizar un estudio macro para ver las constantes de las percepciones de las docentes de educación preescolar con respecto a las prácticas evaluativas y para conocer su punto de vista sobre las estrategias que les permitirían fortalecer sus prácticas evaluativas.

\section{Referencias}

Agut, N. (2010). La evaluación en un modelo de escuela Inclusiva. Innovación educativa. Escuela inclusiva. Aula de Evaluación Educativa, 191, 42-44.

Antúnez, P. A. y Aranguren, R. C. (2004). Problemática teórico-filosófica de la evaluación en la educación básica durante las dos últimas décadas del siglo XX. Educere, 8(25), 149-153

Camilloni R. W. A., Celman, S., Litwin, E. y Palou, M, C. M. (1998). Sistemas de calificación y regímenes de promoción 1. La evaluación de los aprendizajes en el debate didáctico contemporáneo. Buenos Aires: Paidós.

Coll, C. y Onrubia, J. (2002). Evaluar en una escuela para todos. Cuadernos de Pedagogía, 318, 5054. Recuperado de http://www. Psyed.edu.es.grintie

Díaz, B. (2016). Tecnología en secundarias generales. Blog de intercambio de experiencias en el empleo de TIC en ciencias, matemáticas, español, y la asignatura de tecnología en educación secundaria. 
Evaluación docente: ¿̇ la autocrítica del INNE? Recuperado de https://ticsecundariaswordpress.com/

European Agency. (2006). Proyecto IRIS. Evaluación Inclusiva. Improvementt Through research in the inclusive school. Educatión and culture lifelong learning programme comennius. Recuperado de https://educrea.cl/

García, I. y Romero, S. (2016). Avances de la integración educativa/educación inclusiva y la formación docente para la inclusión en México. México: CEMEJUS-UASLP.

Gómez, R. P. y Seda, S. I. (2008). Creencias de las educadoras acerca de la evaluación de sus alumnos preecolares: un estudio de caso. Perfiles Educativos, 30(119), 33-54.

Instituto Nacional de Evaluación de la Educación. (2014). El aprendizaje en preescolar en México. Informe de resultados EXCALE aplicación 2011. Lenguaje y comunicación y pensamiento matemático. México: INEE.

Latorre, A. (2003). La investigación-acción. Conocer y cambiar la práctica educativa. Barcelona: Grao.

Martínez, L. S. E. y Rochera, V. M. (2010). Las prácticas de evaluación de competencias en la educación preescolar mexicana a partir de la reforma curricular. Análisis desde un modelo socioconstructivista y situado. Revista Mexicana de Investigación Educativa, 15(47), 10251050.

Murillo, F. J. y Duck, C. (2012). Una evaluación inclusiva para una educación inclusiva. Revista Latinoamericana de Educación Inclusiva, 6(1), 11-13.

Naciones Unidas (2013). Estudio temático sobre el derecho de las personas con discapacidad a la educación. Recuperado de http://www.acnur.org/t3/fileadmin/Documentos/BDL/

Postic, M. y De Ketelle, J. M. (1992). Observar las situaciones educativas. Madrid: Narcea Ediciones.

Rivas, B. S., Sobrino, M. A. y Peralta, L. F. (2005). La evaluación como garantía de calidad en educación preescolar. Revista Española de Pedagogía, 232, 511-528.

Rivera, F. L y Guerra, M. M. (2005). Retos de la educación preescolar obligatoria en México: la transformación del modelo de supervisión escolar. REICE. Revista Iberoamericana sobre Calidad, Eficacia y Cambio en Educación, 3(1), 503-511.

Santiuste, B. V. y Arranz, M. L. (2008). Nuevas perspectivas en el concepto de evaluación. Revista de Educación, 350, 463-476.

Santos, G. M. A. (1995). La evaluación: Un proceso de diálogo, comprensión y mejora. Granada: Aljibe.

SEP. (2004). Programa de educación preescolar. México: Secretaria de Educación Pública.

SEP. (2010). Experiencias exitosas de integración educativa. Tercera y Cuarta Convocatorias 2007-2008. México: Secretaria de Educación Pública.

SEP. (2011). Programa de Estudio 2011. Guía para la Educadora. Educación Básica Preescolar. México: Secretaria de Educación Pública.

SEP. (2013) Normas generales para la evaluación, acreditación, promoción y certificación en la educación básica. Acuerdo 696. 6 de diciembre de 2013. México: Secretaria de Educación Pública.

Taylor, S. J. y Bodgan, R. (1994). Introducción a los métodos cualitativos en investigación. La búsqueda de los significados. Barcelona: Paidós. 


\section{Breve CV de los autores}

\section{Claudia Isabel Martínez Zapata}

Doctorante de la Benemérita y Centenaria Escuela Normal de San Luis Potosí, Maestra en Psicología por la UASLP, Lic. en Pedagogía por la Universidad Mesoamericana, Lic. en Educación Preescolar por examen CENEVAL. Email: claudiamtz84@yahoo.com.mx

\section{Ismael García Cedillo}

Profesor-Investigador de tiempo completo de la Universidad Autónoma de San Luis Potosí. Miembro del Sistema Nacional de Investigadores. ORCID ID: 0000-0002-48205633. Email: ismaelgace@yahoo.com.mx 